\title{
CMS RPC tracker muon reconstruction
}

This content has been downloaded from IOPscience. Please scroll down to see the full text. 2014 JINST 9 C10027

(http://iopscience.iop.org/1748-0221/9/10/C10027)

View the table of contents for this issue, or go to the journal homepage for more

Download details:

IP Address: 157.193.98.234

This content was downloaded on 11/06/2015 at $14: 03$

Please note that terms and conditions apply. 
$12^{\text {th }}$ Workshop on Resistive Plate Chambers and Related Detectors, 23-28 FEBRUARY 2014,

TSINGHUA UNIVERSITY, BEIJING, CHINA

\section{CMS RPC tracker muon reconstruction}

J. Goh, ${ }^{b, 1}$ M.S. Kim, ${ }^{u}$ Y. Ban, ${ }^{a}$ J. Cai, ${ }^{a}$ Q. Li,${ }^{a}$ S. Liu, ${ }^{a}$ S. Qian, ${ }^{a}$ D. Wang, ${ }^{a}$ Z. Xu, ${ }^{a}$ F. Zhang, ${ }^{a}{ }^{\text {Y. Choi, }}{ }^{b}$ D. Kim, ${ }^{b}$ S. Choi, ${ }^{c}$ B. Hong ${ }_{,}^{c}$ J.W. Kang, ${ }^{c}$ M. Kang ${ }_{,}{ }^{c}$ J.H. Kwon, ${ }^{c}$ K.S. Lee, ${ }^{c}$ S.K. Lee, ${ }^{c}$ S.K. Park, ${ }^{c}$ L.M. Pant, ${ }^{d}$ A.K. Mohanty, ${ }^{d}$ R. Chudasama, ${ }^{d}$ J.B. Singh, ${ }^{e}$ V. Bhatnagar, ${ }^{e}$ A. Mehta, ${ }^{e}$ R. Kumar,${ }^{e}$ S. Cauwenbergh,${ }^{f}$ S. Costantini, ${ }^{f}$ A. Cimmino, ${ }^{f}$ S. Crucy, ${ }^{f}$ A. Fagot,${ }^{f}$ G. Garcia,${ }^{f}$ A. Ocampo, ${ }^{f}$ D. Poyraz,${ }^{f}$ S. Salva,${ }^{f}$ F. Thyssen, ${ }^{f}$ M. Tytgat, ${ }^{f}$ N. Zaganidiss, ${ }^{f}$ W.V. Doninck, ${ }^{g}$ A. Cabrera, ${ }^{h}$ L. Chaparro, ${ }^{h}$ J.P. Gomez, ${ }^{h}$ B. Gomez, ${ }^{h}$ J.C. Sanabria, ${ }^{h}$ C. Avila, ${ }^{h}$ A. Ahmad, ${ }^{i}$ S. Muhammad,${ }^{i}$ M. Shoaib, ${ }^{i}$ H. Hoorani, ${ }^{i}$ I. Awan, ${ }^{i}$ I. Ali, ${ }^{i}$ W. Ahmed, ${ }^{i}$ M.I. Asghar ${ }^{i}$ H. Shahzad,${ }^{i}$ A. Sayed,${ }^{j}$ A. Ibrahim, ${ }^{j}$ S. Ali, ${ }^{j}$ R. Ali, ${ }^{j}$ A. Radi,${ }^{j}$ T. Elkafrawy, ${ }^{j}$ A. Sharma, ${ }^{k}$ S. Colafranceschi, ${ }^{k}$ M. Abbrescia, ${ }^{l}$ C. Calabria, ${ }^{l}$ A. Colaleo, ${ }^{l}$ G. laselli, ${ }^{l}$ F. Loddo, ${ }^{l}$ M. Maggi, ${ }^{l}$ S. Nuzzo, ${ }^{l}$ G. Pugliese, ${ }^{l}$ R. Radogna, ${ }^{l}$ R. Venditti, ${ }^{l}$ P. Verwillingen, ${ }^{l}$ L. Benussi, ${ }^{m}$ S. Bianco, ${ }^{m}$ D. Piccolo, ${ }^{m}$ P. Paolucci,${ }^{n}$ S. Buontempo ${ }^{n}$ N. Cavallo, ${ }^{n}$ M. Merola, ${ }^{n}$ F. Fabozzi, ${ }^{n}$ O.M. Iorio, ${ }^{n}$ A. Braghieri, ${ }^{o}$ P. Montagna, ${ }^{o}$ C. Riccardi, ${ }^{o}$ P. Salvini, ${ }^{o}$ P. Vitulo, ${ }^{o}$ I. Vai, ${ }^{o}$ A. Magnani, ${ }^{o}$ A. Dimitrov, ${ }^{p}$ L. Litov,${ }^{p}$ B. Pavlov, ${ }^{p}$ P. Petkov, ${ }^{p}$ A. Aleksandrov, ${ }^{q}$ V. Genchev, ${ }^{q}$ P. laydjiev, ${ }^{q}$ M. Rodozov, ${ }^{q}$ G. Sultanov, ${ }^{q}$ M. Vutova, ${ }^{q}$ S. Stoykova, ${ }^{q}$ R. Hadjiiska, ${ }^{q}$ H.S. Ibargüen, ${ }^{r}$ M.I.P. Morales, ${ }^{r}$ S.C. Bernardino, ${ }^{r}$ I. Bagaturia, ${ }^{s}$ Z. Tsamalaidze ${ }^{s}$ and I. Crotty ${ }^{t}$ on behalf of the CMS collaboration

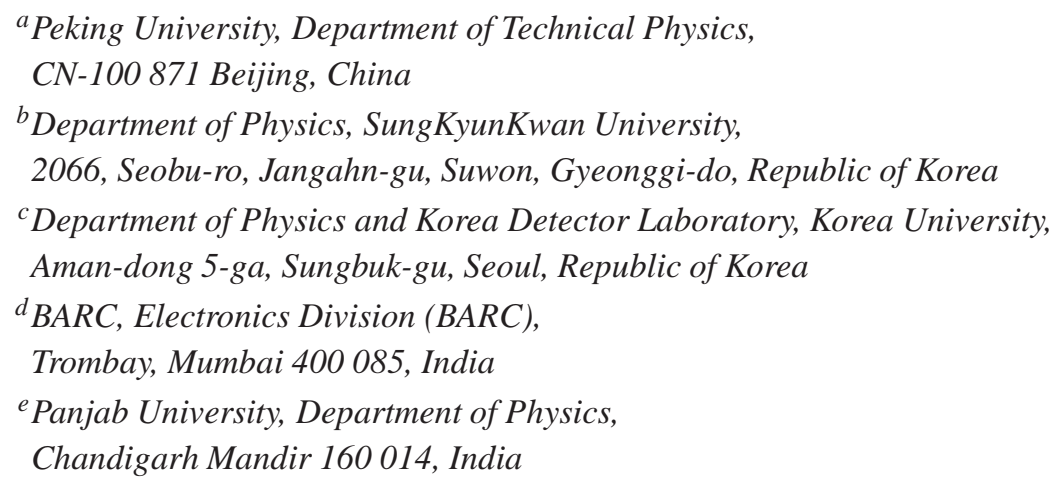

${ }^{1}$ Corresponding author. 
${ }^{f}$ Ghent University, Department of Physics and Astronomy,

Proeftuinstraat 86, BE-9000 Ghent, Belgium

${ }^{g} V U B$, Dienst Elementaire Deeltjes,

Pleinlaan 2 BE-1050 Brussel, Belgium

${ }^{h}$ Universidad de Los Andes, Apartado Aéreo 4976,

Carrera 1E, no. 18A 10, CO-Bogotá, Colombia

${ }^{i}$ Islamabad-NCP, Quaid-I-Azam University,

Islamabad-44000, Pakistan

${ }^{j}$ Academy of Scientific Research and Technology of the Arab Republic of Egypt, 101 Sharia Kasr El-Ain, Cairo, Egypt

${ }^{k} C E R N$,

CH-1211 Geneva 23

${ }^{l}$ Università e INFN, Sezione di Bari,

Via Orabona 4, IT-70126 Bari, Italy

${ }^{m}$ INFN, Laboratori Nazionali di Frascati,

PO Box 13, Via Enrico Fermi 40, IT-00044 Frascati, Italy

${ }^{n}$ Universita e INFN, Sezione di Napoli,

Complesso Univ. Monte S. Angelo, Via Cintia, IT-80126 Napoli, Italy

${ }^{o}$ Università e INFN, Sezione di Pavia,

Via Bassi 6, IT-Pavia, Italy

${ }^{p}$ University of Sofia, Faculty of Physics, Atomic Physics Department,

5, James Bourchier Boulevard, BG-1164 Sofia, Bulgaria

${ }^{q}$ Bulgarian Academy of Sciences, Inst. for Nucl. Res. and Nucl. Energy,

Tzarigradsko shaussee Boulevard 72, BG-1784 Sofia, Bulgaria

${ }^{r}$ Benemérita Universidad Autónoma de Puebla,

Av. San Claudio y 18 sur, edif. 111 A Ciudad Universitaria,

Col. San Manuel, Puebla, Pue. C.P. 72570, Mexico

${ }^{s}$ Tbilisi-IHEPI, Tbilisi State University University,

Street 9, GE-380 086 Tbilisi, Georgia

${ }^{t}$ University of Wisconsin, Department of Physics,

1150 University Avenue Madison, WI 53706, U.S.A.

${ }^{u}$ Kyungpook National University, Department of Physics,

80 Daehakro, Bukgu, Daego, 702-701, Republic of Korea

E-mail: jhgoh@cern.ch

ABSTRACT: A new muon reconstruction algorithm is introduced at the CMS experiment. This algorithm reconstructs muons using only the central tracker and the Resistive Plate Chamber (RPC). The aim of this work is to study how a muon reconstructed only with tracker and RPC information would perform compared to the standard muon reconstruction of the CMS detector. The efficiencies to reconstruct and identify a RPC muon with a transverse momentum greater than $20 \mathrm{GeV} / c$ are measured. The probabilities to misidentify hadrons as muons at low transverse momentum are also reported. These probabilities are compared to the standard muon identification used at CMS.

KeYwORDS: Performance of High Energy Physics Detectors; Resistive-plate chambers 


\section{Contents}

1 Introduction 1

2 Muon reconstruction and identification at CMS 1

3 Efficiency measurement 3

4 Misidentification probability 3

5 Conclusions 4

\section{Introduction}

The Compact Muon Solenoid (CMS) experiment produced important scientific results with the data taken during 2010-2012 runs at $\sqrt{s}=7 \mathrm{TeV}$ and $8 \mathrm{TeV}$, among them the discovery of the Higgs boson [1]. The CMS muon detectors played a central role on several of these results. The CMS muon detectors consist of Drift Tubes (DT), Cathode Strip Chambers (CSC) and Resistive Plate Chambers (RPC) as shown in the figure 1. A complete description of the CMS detector can be found elsewhere [2]. The DTs and CSCs detect muons in the regions $|\eta|<1.2$ and $0.9<|\eta|<2.4$, respectively. The RPCs are installed in both of barrel and endcap regions up to $|\eta|<1.6$ as a complementary muon detector.

The DTs and CSCs provide excellent position and time resolution allowing precise muon tracking in the barrel and endcap region of CMS. Thus RPCs are used as dedicated fast trigger detectors, they complement the triggering capabilities of the DTs and CSCs. The RPC contribution to muon reconstruction has been previously studied using the CMS data taken at $\sqrt{s}=7 \mathrm{TeV}$ [7].

A new muon object called RPC-muon is proposed. The RPC muon object is reconstructed only with the tracker and RPC hits. Three RPC muon identification criteria are defined, their performance is quantified, such as efficiencies and hadron misidentification probabilities using the CMS data taken in 2012 at $\sqrt{s}=8 \mathrm{TeV}$.

\section{Muon reconstruction and identification at CMS}

Muons produced at the center of the CMS detector can leave hits in three or four stations depending on their pseudo-rapidity. Muons are reconstructed using the hits in the muon detectors and tracks in the silicon inner tracker. Three types of muon reconstruction algorithms are used in CMS. On the muon system, tracking can be done using only track segments reconstructed with DT and CSC hits, these tracks are identified as muons are called standalone muons. Tracker muons are reconstructed with inner tracks matched to muon segments, the matching is done after extrapolating the inner tracks up to the muon stations. Global muons are reconstructed combining inner tracks 


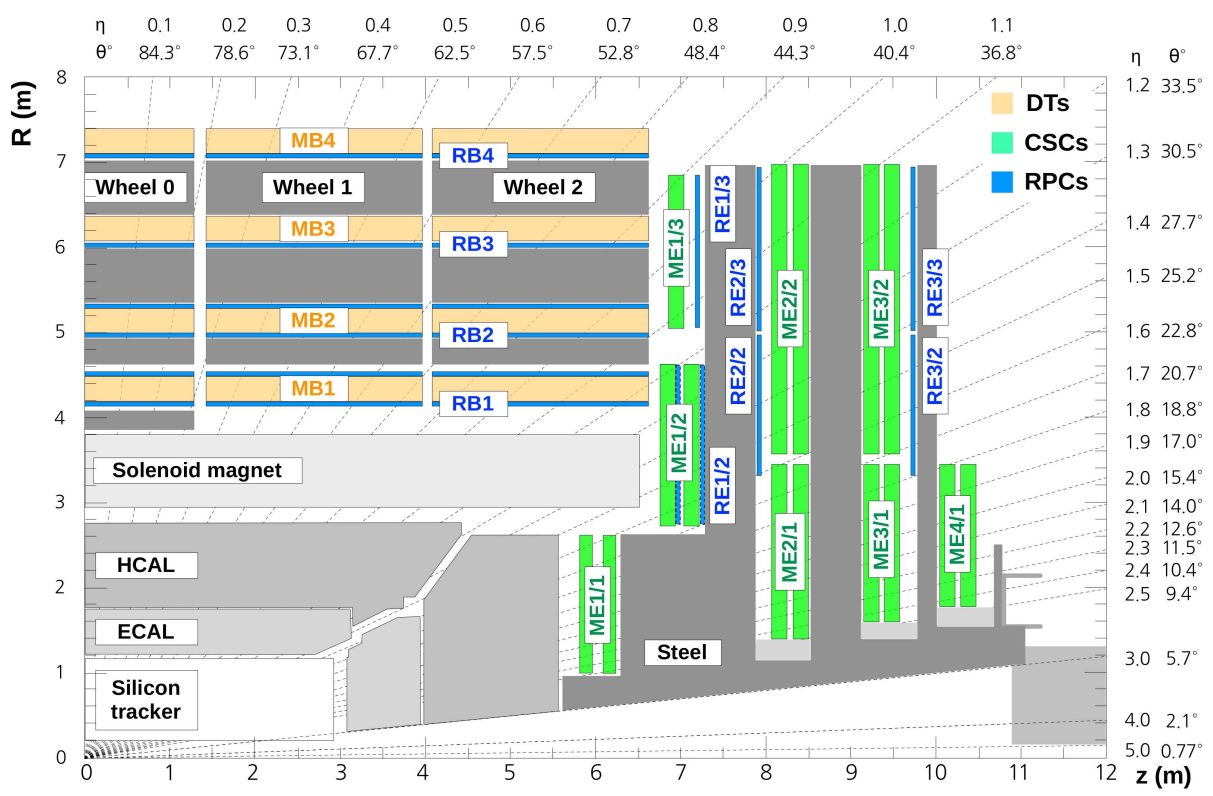

Figure 1. Layout of one quadrant of CMS. The figure shows the four DT stations in the barrel (MB1-MB4, yellow), the four CSC stations in the endcap (ME1-ME4, green), and the RPC stations (RB1-RB4 and RE1-RE3).

and standalone muon tracks with full global track fit. In addition, particle flow algorithm [3] is applied to reconstruct muons without ambiguity [4].

There are two major muon identification algorithms in CMS, loose muons and tight muons. Loose muons are particles identified as muons with particle flow algorithm. A muon candidate is discarded from the loose muons if it is reconstructed without inner tracker information. Tight muons are global muons which are also identified with the particle flow algorithm. At least one muon chamber hit must be used in the global track fit with $\chi^{2} / n d f<10$. Also, at least two muon segments have to be matched when the inner track is extrapolated to the muon stations. Hits on more than 5 layers of the inner tracker and at least one pixel hit are required. Finally, cuts on the impact parameters in the transverse and longitudinal planes with respect to the primary vertex of the event are required to be $d_{\mathrm{xy}}<0.2 \mathrm{~cm}$ and $d_{\mathrm{z}}<0.5 \mathrm{~cm} \mathrm{[4]}$.

There are additional muon identification criteria based on tracker muons, depending on association of muon hits or segments to tracks. The tracker muon identifications are expected to recover some muons when some chambers are not efficient or missing along their trajectory.

The RPC muons are defined as a subset of the tracker muons. First, tracks reconstructed with the inner tracker are extrapolated to muon stations using the Kalmann filter algorithm [6]. A RPC hit is associated with the track, if the extrapolated point is within $\Delta x<20 \mathrm{~cm}$ and $\Delta x / \sigma x<4$ from the RPC local $x$-coordinate. Three different RPC-muon working points are defined corresponding to the number of RPC layers or stations with associated RPC hits. The first working point is called RPCMuLoose, it requires at least two RPC layers with associated RPC hits. RPCMuMedium working point is defined by requiring the associated RPC layers to be in different stations. For the tightest RPC-muon identification, RPCMuTight, at least three RPC layers with associated RPC hits are required. 

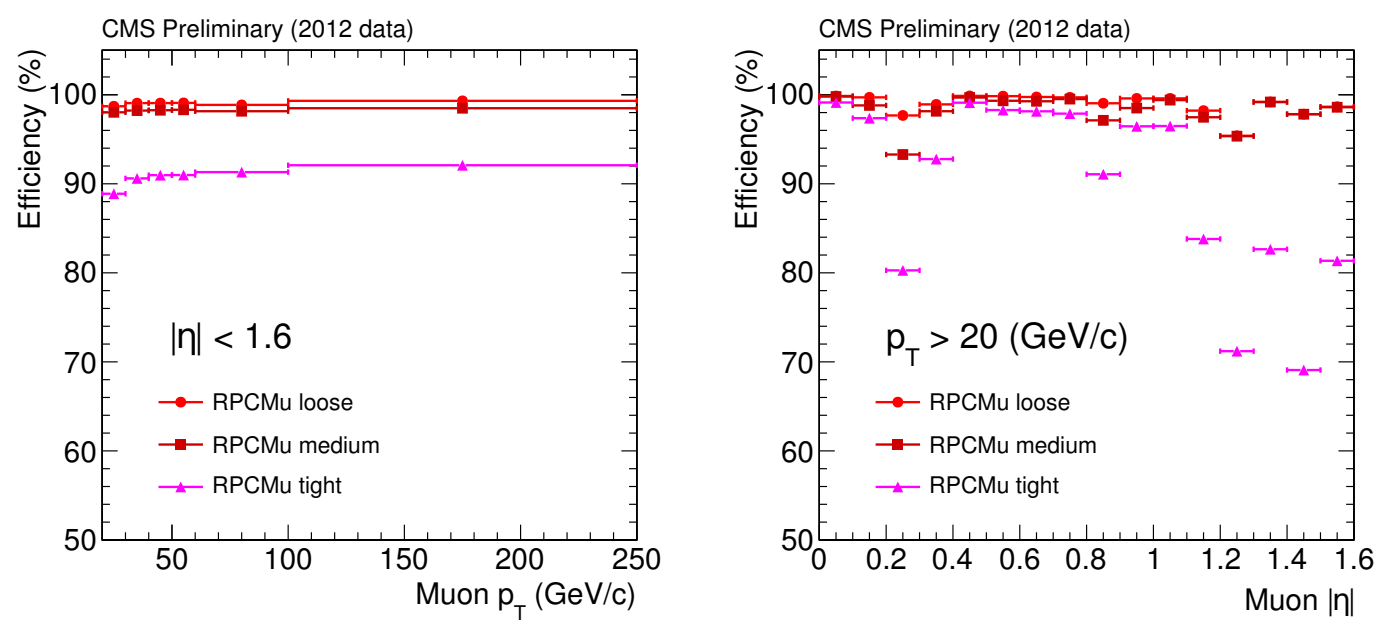

Figure 2. Efficiency of high $p_{T}$ RPC muon working points for general tracks with basic quality cuts in RPC coverage. Efficiencies are shown as a function of $p_{T}$ (left) and $|\eta|$ (right).

\section{Efficiency measurement}

In this study, the efficiencies of RPC muon identification criteria are reported for muons with $p_{T}>20 \mathrm{GeV} / c$ and $|\eta|<1.6$. The muons used in the analysis come from the decay of $Z^{0}$ bosons mostly. The events used were taken during the 2012 run, they were triggered on single muons, the total integrated luminosity used is $19.6 \mathrm{fb}^{-1}$. A high purity and unbiased muon sample is guaranteed by requiring a tight muon identification on the leading muon coming from $Z^{0}$ boson, this identified muons are labeled tag muon. To avoid trigger bias, the tag is required to be the triggered muon. The another leg is a probe used to measure the efficiency. No identification criteria was required on the probe with the exception of minimum requirements that guaranteed the track quality in the inner tracker where at least 10 valid hits in the inner tracker, and impact parameter cuts with respect to the primary vertex, $d_{\mathrm{xy}}<0.2 \mathrm{~cm}$ and $d_{\mathrm{z}}<0.5 \mathrm{~cm}$.

To extract the efficiency from the data, simultaneous binned likelihood fits were performed, the fits were done on the ratio between $Z^{0}$ candidates with probes passing the identification criteria, and all of the candidates. $Z^{0}$ signal is modeled as a Breit-Wigner function convoluted with a gaussian resolution and a background distribution taken as a 2 nd order polynomial.

The efficiency of high $p_{T}$ RPCMuon is measured to be more than $98 \%$ for RPCMuLoose and RPCMuMedium as shown in the figure 2. There are efficiency drops in $|\eta|=0.2,0.8$ and $|\eta|>1$ which reflect the detector layout. Efficiency drops in the barrel correspond to the spaces between wheels. The efficiency drops at high pseudorapidity region are visible for the RPCMuTight due to the fact that there are only 3 RPC layers in the endcaps as shown in the figure 1.

\section{Misidentification probability}

Some hadrons can be misreconstructed as muons during the identification. For example, a pion or kaon can decay into a muon and neutrinos. The secondary muon hit can be linked with the hadron track and identified as muons (decay-in-flight). Some hadrons can pass through the calorimeters and propagate to the muon detectors (punch-through). If tracks multiplicity is very high, a 


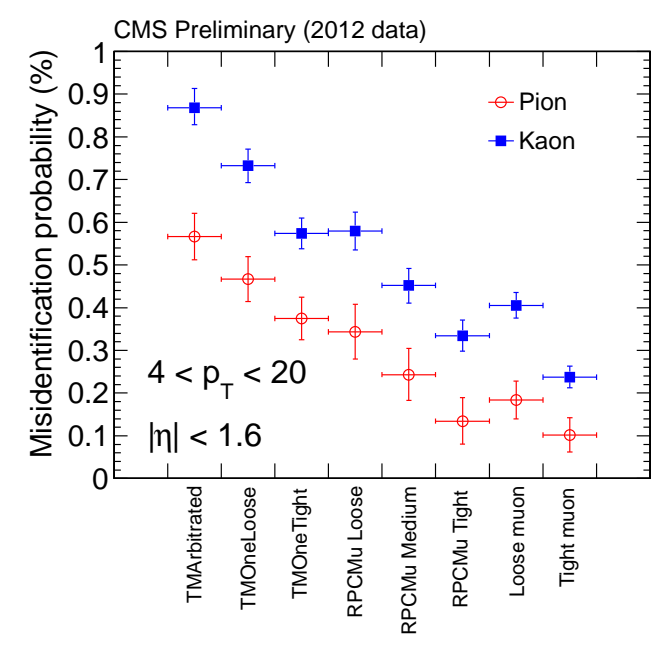

Figure 3. Muon misidentification probability.

pion track can be matched with muon hits by chance (random matching). The misidentification probabilities of hadrons were measured using the well-known decays of resonances. For the pion misidentification probability, the decay of $K_{s}^{0}$ meson into pion pairs was used. In a similar way, $\phi^{0}$ meson were used to study kaon misidentification probability.

The misidentification probability of RPCMuLoose working point is measured to be $0.364 \pm$ $0.073 \%$ for pions and $0.579 \pm 0.045 \%$ for kaons which is comparable to the tracker muon identification with one station requirement. The medium working point of RPCMuon identification shows a lower misidentification probability, reaching to $0.261 \pm 0.069 \%$ (pion) and $0.452 \pm 0.041 \%$ (kaon). Overall misidentification probabilities for the tracker muons, RPCMuons and the standard muon identification in CMS are shown in the figure 3. The misidentification probability of RPCMuMedium is comparable to one for loose muon, which is measured to be $0.171 \pm 0.050 \%$ (pion) and $0.405 \pm 0.030 \%$ (kaon). RPCMuTight shows best performance among the three RPCMuon identifications where its values are $0.147 \pm 0.063 \%$ (pion) and $0.335 \pm 0.036 \%$ (kaon). Dependencies of misidentification probabilities to the hadron transverse momentum and pseudorapidities are also presented in the figure 4 and 5 .

\section{Conclusions}

The muon reconstruction with RPC hits was implemented as one of the standard algorithms of the CMS experiment. Efficiencies of RPC tracker muon reconstruction algorithms were measured using the Tag-and-probe technique in the region of transverse momentum above $4 \mathrm{GeV} / c$ and pseudorapidity $|\eta|<1.6$ using muons from the decay of $Z^{0}$ bosons. The efficiencies of loose and medium working points of RPC tracker muon algorithms were measured to be greater than $98 \%$. The misidentification probabilities from hadrons were measured using the decay of $K_{s}^{0}$ and $\phi^{0}$ mesons. The $\pi^{ \pm} \rightarrow \mu^{ \pm}$misidentification probability at the loose working point is $0.364 \pm 0.073 \%$ and the equivalent number for $K^{ \pm} \rightarrow \mu^{ \pm}$is $0.579 \pm 0.045 \%$ which is comparable to the standard identification criteria. 

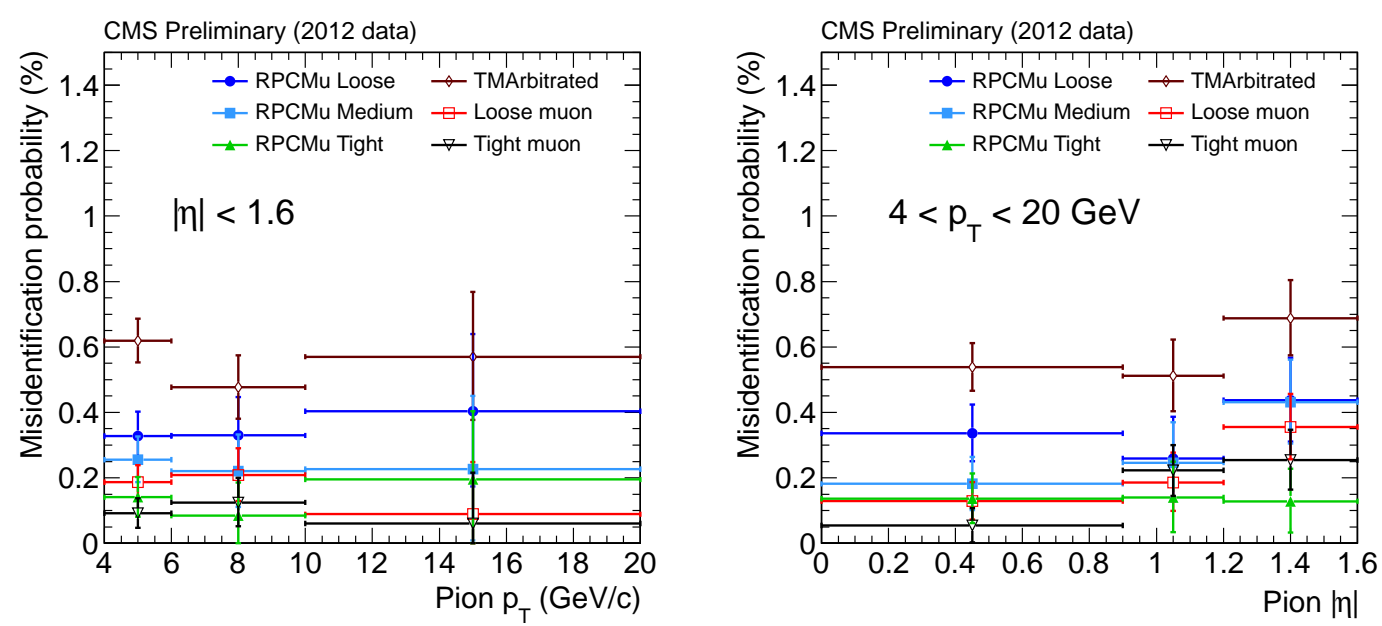

Figure 4. Muon misidentification probability from pions in transverse momentum and $|\eta|$.
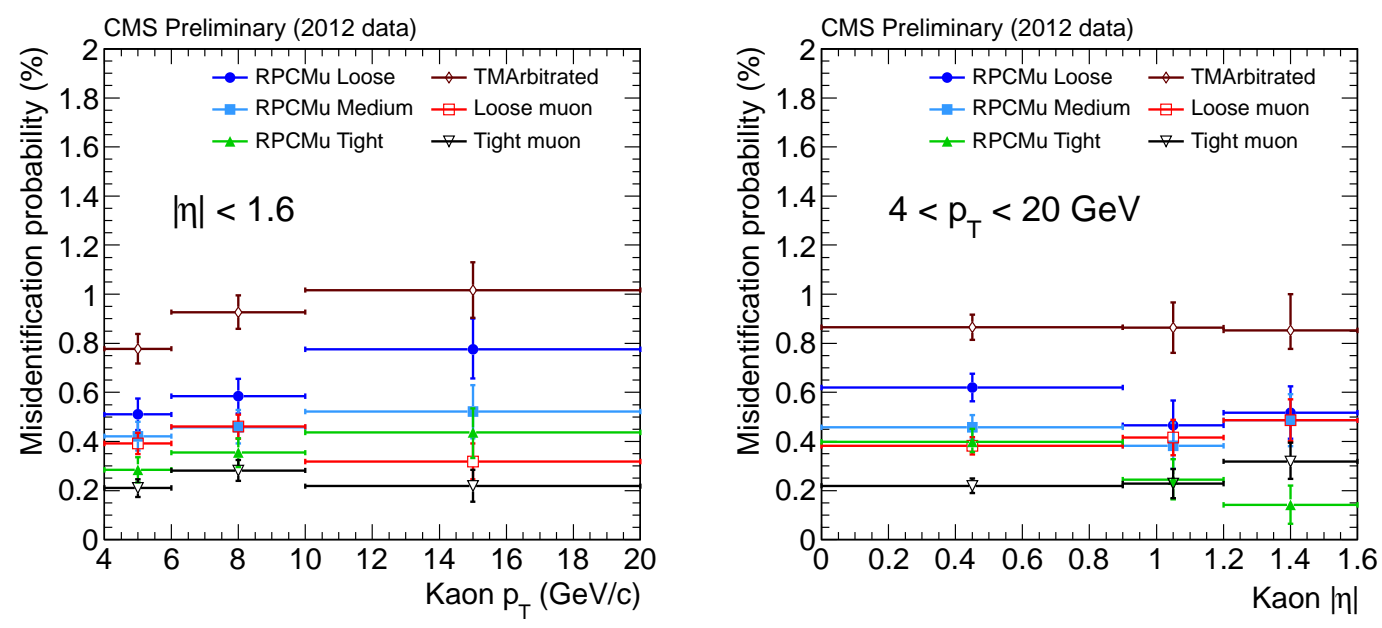

Figure 5. Muon misidentification probability from kaons in transverse momentum and $|\eta|$.

\section{Acknowledgments}

We congratulate our colleagues in the CERN accelerator departments for the excellent performance of the LHC and thank the technical and administrative staffs at CERN and at other CMS institutes for their contributions to the success of the CMS effort. In addition, we gratefully acknowledge the computing centres and personnel of the Worldwide LHC Computing Grid for delivering so effectively the computing infrastructure essential to our analyses. Finally, we acknowledge the enduring support for the construction and operation of the LHC and the CMS detector provided by the following funding agencies: BMWFW and FWF (Austria); FNRS and FWO (Belgium); CNPq, CAPES, FAPERJ, and FAPESP (Brazil); MES (Bulgaria); CERN; CAS, MoST, and NSFC (China); COLCIENCIAS (Colombia); MSES and CSF (Croatia); RPF (Cyprus); MoER, ERC IUT and ERDF (Estonia); Academy of Finland, MEC, and HIP (Finland); CEA and CNRS/IN2P3 
(France); BMBF, DFG, and HGF (Germany); GSRT (Greece); OTKA and NIH (Hungary); DAE and DST (India); IPM (Iran); SFI (Ireland); INFN (Italy); NRF and WCU (Republic of Korea); LAS (Lithuania); MOE and UM (Malaysia); CINVESTAV, CONACYT, SEP, and UASLP-FAI (Mexico); MBIE (New Zealand); PAEC (Pakistan); MSHE and NSC (Poland); FCT (Portugal); JINR (Dubna); MON, RosAtom, RAS and RFBR (Russia); MESTD (Serbia); SEIDI and CPAN (Spain); Swiss Funding Agencies (Switzerland); MST (Taipei); ThEPCenter, IPST, STAR and NSTDA (Thailand); TUBITAK and TAEK (Turkey); NASU and SFFR (Ukraine); STFC (United Kingdom); DOE and NSF (U.S.A.).

\section{References}

[1] CMS collaboration, Observation of a new boson at a mass of $125 \mathrm{GeV}$ with the CMS experiment at the LHC, Phys. Lett. B 716 (2012) 30 [arXiv: 1207.7235].

[2] CMS collaboration, The CMS experiment at the CERN LHC, 2008 JINST 3 S08004.

[3] CMS collaboration, Particle-Flow Event Reconstruction in CMS and Performance for Jets, Taus and MET, CMS-PAS-PFT-09-001.

[4] CMS collaboration, Performance of CMS muon reconstruction in pp collision events at $\sqrt{s}=7 \mathrm{TeV}$, 2012 JINST 7 P10002 [arXiv:1206.4071].

[5] CMS collaboration, Performance Study of the CMS Barrel Resistive Plate Chambers with Cosmic Rays, 2010 JINST 5 T03017 [arXiv:0911.4045].

[6] R. Fruhwirth, Application of Kalman filtering to track and vertex fitting, Nucl. Instrum. Meth. A 262 (1987) 444 [HEPHY-PUB-87-503].

[7] CMS collaboration, CMS reconstruction improvement for the muon tracking by the RPC chambers, 2013 JINST 8 T03001 [arXiv: 1209.2646]. 